\title{
Germanica
}

contemporain : jeux intermédiaux, modes de transfert, adaptations

\section{Traces d'un effacement et effacement des traces : La Tour, du roman au film}

Traces of effacement and effacement of traces: the television adaptation of the novel Der Turm

Spuren der Auslöschung und Auslöschung der Spuren - die Verfilmung des

Romans Der Turm

Éric Leroy du Cardonnoy

\section{(2) OpenEdition}

Édition électronique

URL : http://journals.openedition.org/germanica/2291

DOI : 10.4000/germanica.2291

ISSN : 2107-0784

Éditeur

Université de Lille

Édition imprimée

Date de publication : 30 décembre 2013

Pagination : $211-224$

ISBN : 9782913857322

ISSN : 0984-2632

Référence électronique

Éric Leroy du Cardonnoy, "Traces d'un effacement et effacement des traces : La Tour, du roman au film », Germanica [En ligne], 53 | 2013, mis en ligne le 30 décembre 2016, consulté le 06 octobre 2020. URL : http://journals.openedition.org/germanica/2291; DOI : https://doi.org/10.4000/germanica.2291

(c) Tous droits réservés 


\title{
Traces d'un effacement et effacement des traces : La Tour, du roman au film
}

\author{
Éric LEROY DU CARDONNOY \\ Université de Caen Basse-Normandie \\ C'est peut-être l'exercice artistique du futur : \\ $[\ldots]$ refaire quelque chose qui transforme ce \\ qui a été en quelque chose de secrètement \\ nouveau... 1
}

Comme bien souvent de nos jours, un roman, devenu un bestseller, se voit adapté à la télévision, le succès de librairie devant par avance garantir le succès de l'adaptation. Ainsi le film La Tour d'Uwe Tellkamp, paru en 2008 , et qui « malgré » ses quelque mille pages a rapidement escaladé l'échelle des ventes pour devenir un grand succès - il a reçu le Prix du Livre allemand l'année de sa parution -, a été en 2012 diffusé, pour la première partie, sur la première chaîne allemande nationale, ARD, le 3 octobre, jour de l'unité allemande (Tag der deutschen Einheit), c'est-à-dire le jour de la fête nationale qui marque la disparition de la RDA et célèbre la réunification. Après une mise au point sur les conditions de projection du téléfilm qui en déterminent le pacte de lecture, nous considérerons la question théorique de l'adaptation d'une œuvre romanesque à la télévision. Puis nous nous intéresserons, à partir des recensions critiques faisant suite à la diffusion du téléfilm, aux différences fondamentales qui apparaissent entre l'adaptation

1. - Citation tirée du roman cité par Bernard Umbrecht, « Un pays évanoui. $L a$ Tour d'Uwe Tellkamp », in : Le Monde diplomatique, Littérature du monde, mai 2012. 
et le roman pour en comprendre les conséquences en nous concentrant sur trois points en particulier : tout d'abord une comparaison entre l'incipit du roman et celui du téléfilm, puis une interrogation sur la coupure effectuée entre les deux parties du téléfilm, enfin le recentrage de cette production par rapport au foisonnement polyphonique du roman. En d'autres termes, les choix opérés par le réalisateur et le scénariste retiendront notre attention pour comprendre la portée de leur travail.

\section{Pacte de lecture et questions sur ce que le cinéma fait à la littérature}

Le téléfilm La Tour (scénario : Thomas Kirchner, réalisation : Christian Schwochow) ne peut être regardé, interprété ni compris sans qu'il soit tenu compte des conditions particulières dans lesquelles il a été projeté. En effet, c'est le 3 octobre, jour de la fête nationale allemande, qu'il a été diffusé pour la première fois en exclusivité sur la première chaîne généraliste de la télévision publique, à une heure de grande écoute - on dirait en jargon télévisuel en « prime time »-, c'està-dire à $20 \mathrm{~h} 15$. Le choix du jour n'est bien évidemment pas anodin, mais relève d'une intention très claire qui est de faire du téléfilm un document pour célébrer l'événement historique du jour. Il fut suivi à $21 \mathrm{~h} 45$ d'un documentaire, signé Jan N. Lorenzen, s'interrogeant sur " la manière dont était la vie dans la réalité, dont les Allemands de l'Est ont vécu les années 80 et sur le rôle particulier de Dresde ${ }^{2}$. $\gg$ Mais il avait également été précédé par toute une série d'émissions télévisées et radiophoniques préparatoires dans les semaines antérieures à sa diffusion (rencontre avec l'auteur, adaptation radiophonique de certains passages du roman, discussions et tables rondes sur la vie en RDA dans les années 80, entretiens avec les acteurs du film³ ${ }^{3}$ etc.) ; la chaîne MDR, offrant le 3 octobre au soir la possibilité de le regarder parallèlement en streaming, avait ouvert un blog et un compte Twitter pour recueillir les commentaires et les questions des téléspectateurs auxquelles répondit le réalisateur ${ }^{4}$. Il fut regardé par environ sept millions de personnes. Le producteur principal, MDR, chaîne de radio et télévision couvrant respectivement la Saxe, le Saxe-Anhalt et la Thuringe, a donc réussi, par

2. - «Wie dieses Leben in der Realität aussah, wie die Menschen im Osten Deutschlands die 80er-Jahre erlebten und welche besondere Rolle Dresden spielte, diesen Fragen ging im Anschluss an den ersten Teil des Films am 3. Oktober eine MDRDokumentation von Jan N. Lorenzen nach. » in : MDR-Jahresbericht 2012, p. 23.

3. - On rappellera ici que la plupart des acteurs du téléfilm sont des anciens Allemands de l'Est, ce qui, de l'aveu de la chaîne (Idem, p. 23 : « konnten Lebenserfahrungen aus DDR-Zeiten einbringen »), n'a pu que contribuer au succès de l'entreprise dans une visée d'authenticité.

4. - Idem, p. 24. 
un battage médiatique de plusieurs semaines, à préparer le public à une certaine forme de réception du téléfilm en deux parties. Le fait d'avoir choisi un jour historique si fortement symbolique pour sa première projection officielle n'a rien du hasard : il s'agit bien d'orienter vers une « lecture » historique, naturaliste et réaliste du téléfilm et de considérer la narration du film comme une œuvre de témoignage presqu'à la manière d'un documentaire historique. Tout cela peut expliquer, entre autres, pourquoi il a obtenu le Publikums-Bambi (2012) et le GrimmePreis pour la fiction (2013), deux récompenses prestigieuses de la télévision allemande ${ }^{5}$. Ainsi La Tour d'Uwe Tellkamp, le roman comme le téléfilm, est présentée et attendue comme la fresque d'une époque disparue, comme le roman d'un monde révolu dont il s'agirait à jamais de conserver la ou les traces. Cependant, comme l'écrit Jacques Derrida,

Méditer l'écriture, c'est-à-dire aussi bien l'effacement - et la production d'écriture est aussi la production d'un système d'effacement, la trace est à la fois ce qui s'inscrit et ce qui s'efface - , c'est méditer constamment ce qui rend illisible ou ce qui est rendu illisible ${ }^{6}$.

Ainsi Uwe Tellkamp donne à lire ce que fut la RDA, mais dans le même temps, par cette réflexion sur ce qui s'efface et disparaît, il produit le système même d'effacement de ce qu'il convoque en le rendant illisible, voire invisible : il transforme une réalité en fiction. En effet, lire $L a$ Tour, c'est regarder, lire un texte qui, en recréant ce qui a disparu, l'efface une seconde fois et le rend doublement illisible. D'une part, il y va d'une production d'écriture dans un processus qu'on exige anamnétique (la recherche des traces), d'autre part, cette production doit de manière tout à fait redondante et paradoxale mettre au jour le système d'effacement propre à toute écriture, et spécialement à la sienne, par la recréation fantastique et romanesque d'un univers. Il y va donc bien des traces, de la recherche des traces, pour la plupart involontairement effacées, qu'il s'agit de faire réapparaître, comme le chasseur, puisque la trace s'inscrit dans le champ d'une métaphorique cynégétique. À bien des égards, le roman s'apparente à une entreprise située à la limite de la contradiction, de l'impossible, de l'impensable, et dans le même temps à une reconnaissance de dette à l'égard de tout ce qui a participé à son élaboration.

Que le roman se termine sur les grandes manifestations de Dresde en 1989 lors du passage du train des " fugitifs de la République » (Republikflüchtlinge) en transit de la Tchécoslovaquie vers l'Allemagne

5. - La chaîne MDR a également ouvert une page web entièrement dédiée au téléfilm : http://www.mdr.de/der-turm/index.html (page consultée pour la dernière fois le 30 septembre 2013).

6. - Jacques Derrida, Points de suspension, Paris, Galilée, 1998, p. 403. 
de l'Ouest, voilà qui fixe un point de non-retour pour la RDA et indique une date bien précise.

Une date inscrit cette singularité dans une lisibilité, c'est-à-dire en référence à un calendrier, à des marques en tout cas répétables, qui sont accessibles à tout le monde ${ }^{7}$.

Les textes littéraires ne peuvent pas être un simple « reflet », mais sont toujours une réponse à une situation et à un état particulier et production d'un nouveau sens qui déplace ou sanctifie les positions à l'intérieur du champ en question. Il ne s'agit donc pas seulement de la question d'une adaptation, mais d'une fiction dans laquelle il faudra reconnaître, identifier une réalité telle qu'on l'a déjà vue, par exemple, dans La Vie des autres, Good-bye Lenin ou encore Sonnenallee, pour ne citer que les films les plus connus traitant de l'ex-RDA. Cela signifie également, par conséquent, que ce roman adapté pour la télévision est loin d'être une œuvre unique, qu'elle coexiste avec d'autres productions du même type parmi lesquelles elle doit se frayer une place, investir une part du champ cinématographique pour s'imposer et se faire un nom. Enfin, comme Gerd Röllecke l'avait fait remarquer dans sa recension pour la $F A Z$,

La vérité au sujet de la RDA ? Le roman n'est pas un livre d'histoire. «L'action de ce roman est le libre fruit de l'imagination. »C'est une œuvre d'art, un tableau. Mais les œuvres d'art ont leur vérité intérieure qui montre parfois plus qu'une photographie précise ${ }^{8}$.

Il ressort de cette remarque du critique, faite avant que les droits d'adaptation aient été vendus, qu'il avait déjà perçu le danger d'une «lecture » possible du roman : le considérer d'un point de vue strictement historique, voire historiographique, et comme un témoignage ethnographique et sociologique " d'un pays disparu » (comme l'indique le sous-titre du roman; en traduction française : « d'une terre engloutie »). Autrement dit, il s'agit d'une lecture réductrice qui ne perçoit pas la nature spécifique de la littérature qui, bien qu'elle soit également un témoignage, est aussi, précisément, une œuvre d'art avec ses règles, notamment du fait qu'elle n'est pas simplement dénotative. D'autant que l'auteur a bien pris soin d'indiquer par le soustitre de l'œuvre qu'il s'agit d'une « histoire».

Il va de soi que nous ne nous attacherons pas à attribuer un satisfecit ou non à la fidélité, la trahison ou l'appauvrissement de l'adaptation

7. - Idem, p. 390-391.

8. - « Die Wahrheit über die DDR? Der Roman ist kein Geschichtswerk. "Die Handlung ist frei erfunden." Er ist ein Kunstwerk, ein Gemälde. Aber Kunstwerke haben ihre innere Wahrheit, und die zeigt manchmal mehr als eine scharfe Fotografie. » Gerd Röllecke, « Tellkamps Turm. Ein Bürgertum ohne Politik ? », FAZ, 26/09/2010. 
filmique, ce qui relèverait de l'illusionnisme naturaliste. En revanche, l'examen des choix opérés par le scénariste et le réalisateur sera d'autant plus intéressant qu'il permettra de comprendre ce qu'ils ont voulu en faire et quelle en a été la réception dans le cadre que nous venons d'esquisser ${ }^{9}$. Adapter un film au cinéma ou, comme dans le cas qui nous occupe, à la télévision ne signifie pas uniquement réécrire, mais avant tout faire passer d'un langage à un autre, d'un média à un autre, d'un langage à des langages ; l'image est bien entendu primordiale, mais il ne faut pas oublier la bande-son, l'organisation des séquences ainsi que la perception, la sélection, la recomposition ordonnées par le spectateur. Comme Francis Vanoye l'explique dans L'Adaptation littéraire au cinéma ${ }^{10}$, l'adaptation d'une œuvre littéraire à l'écran est toujours à envisager sous l'angle du travail (travail comme douleur, travail du rêve, travail du bois) qui fait que l'adaptation est d'emblée toujours suspecte, de vouloir par exemple se passer de l'œuvre-source, et «place d'emblée le cinéma en posture de dépendance et de dette par rapport à la littérature ${ }^{11} »$. En effet se trouvent posées des questions de réflexion, tant théoriques que pratiques, telles que plagiat, valorisation/ dévalorisation, auteur et son rôle, le scénariste et le réalisateur, masque, traduction, etc. Michel Serceau explique l'histoire des relations entre cinéma et littérature et surtout la longue liste des griefs de la seconde à l'encontre du premier, liste qui repose, selon lui, sur « un clivage entre deux démarches : l'une qui réduit le film à sa structure narrative, l'autre qui mue la représentation en écriture ${ }^{12}$ ». L'adaptation, en effet, «n'est pas seulement une opération factuelle - traduction d'un langage dans un autre - qui placerait le cinéma dans la dépendance de la littérature et par laquelle il se ravalerait au rang d'illustrateur, mais un faisceau de

9. - « La répartition typologique des adaptations d'œuvres littéraires à l'écran pourrait relever, en première approximation, du choix fait par le réalisateur entre deux partis aisément identifiables : un parti de transposition extrême - qu'on pourrait appeler de réécriture si la réécriture n'était, de toute façon, imposée par le changement de la substance de l'expression - et un parti de proximité, expression évidemment métaphorique permettant d'éviter d'une part le mot d'illustration qui, à juste titre, marque le défaut d'invention, d'autre part ceux de fidélité ou de respect qui imposent d'emblée une hiérarchie en faveur de l'œuvre d'origine. » Jean Lelaidier, « Gens de dublin : le film en deçà/au delà du texte ", in : La Licorne. Revue de langue et de littérature françaises. Les Cahiers en ligne : littératures et esthétique, août 2005. http://licorne.edel.univ-poitiers. fr/document.php?id=385 (page consultée le 28 juillet 2013 .

10. - Francis Vanoye, L'adaptation littéraire au cinéma : formes, usages, problèmes, Paris, Armand Colin, 2011, avant-propos, p. 6.

11. - Idem, p. 12.

12. - Michel Serceau, L'Adaptation cinématographique des textes littéraires. Théories et lectures, Liège, éd. du CEFAL, 1999, coll. « Grand écran petit écran » Essais, p. 7. 
réalités sémiotiques et esthétiques ${ }^{13}{ }$. L'adaptation, réalité plurielle et réversible, est un mode de réception et d'interprétation qui pose la question de «l'articulation, du retour, de l'obsolescence ou de la pérennité des différentes formes de représentation ${ }^{14} \gg$ où se structure une part non négligeable de l'imaginaire de l'homme d'aujourd'hui et, dans le cas qui nous occupe, celui du citoyen allemand en 2012. Il s'agit plutôt d'une « re-création », comme l'explique Robert Stam :

On peut considérer l'adaptation comme la transformation de l'hypotexte d'un roman source à travers une série complexe d'opérations : sélection, amplification, concrétisation, actualisation, critique, extrapolation, analogie, popularisation et ré-acculturation ${ }^{15}$.

Car, comme l'écrit Mathilde Labbé à propos de deux adaptations de Boule de Suif de Maupassant :

Le passage de l'écrit à l'audiovisuel fixe dans la matérialité des personnages des décors et des sons qui n'étaient que mots. De ce fait, l'histoire prend une nouvelle dimension : l'image qui s'impose aux spectateurs devient le seul référent pictural possible (alors que chacun s'imagine le personnage à sa manière pendant la lecture) et s'inscrit dans une histoire commune des souvenirs cinématographiques ${ }^{16}$.

On l'aura compris, la situation particulière, pour ne pas dire exceptionnelle, de projection du téléfilm a bien pour objectif d'inscrire les images du film dans une histoire commune et de créer des souvenirs non cinématographiques dans une histoire collective. Cependant, nous pouvons dire que ce désir d'orientation de la réception est aussi déterminé par le genre auquel appartient cette production. Le genre est en effet le moyen d'établir avec le destinataire « un contrat de lecture qui encadre son attitude de réception mais aussi, rétrospectivement, le travail de production des messages émis ${ }^{17} \gg$. Stéphane Benassi distingue ainsi trois genres principaux : le téléfilm, le feuilleton et la série comme les trois formes naturelles de la fiction télévisuelle pour

13. - Idem, p. 9 .

14. - Idem, p. 10.

15. - « One way to look at adaptation is to see it as a matter of a source novel's hypotext being transformed by a complex series of operations ; selection, amplification, concretization, actualization, critique, extrapolation, analogization, popularization, and reculturalization. » Robert Stam, « Beyond Fidelity : The Dialogics of Adaptation », Film Adaptation, ed by James Naremore, New Brunswick-New Jersey, Rutgers UP, 2000, p. 68-69.

16. - Mathilde Labbé, « Ce que le cinéma fait à Boule de suif », p. $6:$ in $L H T \mathrm{n}^{\circ} 2$, «Ce que le cinéma fait à la littérature (et réciproquement) », décembre 2006. URL : http://www.fabula.org/lht/2/index.php?id=252 consulté le 15 juillet 2013.

17. - Stéphane Benassi, Séries et feuilletons T.V.: pour une typologie des fictions télévisuelles, Liège, éd. du CEFAL, 2000, coll. «Grand écran petit écran » Essais, p. 8. 
conclure que la fiction télévisuelle serait devenue « un compromis entre roman et cinéma ${ }^{18}$ ». Comme nous l'avons rappelé plus haut, le téléfilm a été présenté sur deux jours, donc il ne sera pas question de feuilletonisation, opération de dilatation et complexification de la diégèse. Cependant, les deux fois $1 \mathrm{~h} 30 \mathrm{du}$ téléfilm devront retenir l'attention afin de comprendre et d'interpréter les raisons qui ont pu présider au choix d'une telle organisation et du découpage de la diégèse, autrement dit les raisons et les conditions de la reconfiguration du récit, sachant que, comme le pense François Jost à la différence de John Searle, l'intentionnalité est peut-être plus à chercher du côté du destinataire en tant que construction du spectateur que du côté de l'émetteur.

\section{Incipit, recentrage et coupure}

Uwe Tellkamp a placé en exergue de son œuvre deux phrases qui la positionnent dans le champ littéraire en l'insérant dans une catégorie générique qui est celle du roman et précise :

L'action de ce roman est le libre fruit de l'imagination. Les personnages, tels qu'ils sont décrits, vivent dans la représentation et ont autant de points communs avec des êtres réels que l'argile du plasticien avec la sculpture ${ }^{19}$.

Le protocole de lecture que nous propose Uwe Tellkamp est donc très clair : même s'il n'est pas possible de nier le rapport de l'action du roman à une réalité référentielle, elle ne peut être considérée comme une illustration de celle-ci. Recourant à un art figuratif, la sculpture, il indique que la matière première du roman est bien réelle, mais que le jeu de la création artistique réside précisément dans ce que le résultat final a subi un traitement de raffinement, d'esthétisation qui ne permet plus de la reconnaître. L'art en général et la littérature en particulier sont par conséquent formation, reformation, déformation d'une réalité qui, comme le rappelait Jacques Derrida, efface les traces de sa création. Autrement dit, écrire comme Jens Binsky dans sa recension pour la Süddeutsche Zeitung citée en quatrième de couverture :

À qui voudra à l'avenir savoir comment la vie s'est réellement passée dans la RDA finissante, il faudra rapidement et de manière décidée lui mettre le nouveau roman d'Uwe Tellkamp dans les mains ${ }^{20}$.

18. - Idem., p. 42.

19. - « Die Handlung dieses Romans ist frei erfunden. Die Personen, wie sie geschildert werden, leben in der Vorstellung und haben mit tatsächlich existierenden Menschen soviel gemein wie der Bildhauerton mit einer Skulptur. » Uwe Tellkamp, Der Turm, Frankfurt/ Main, Suhrkamp, 2008, p. 4 (traduction française Olivier Mannoni, Paris, Grasset, 2012, p. 7).

20. - « Wenn in Zukunft einer wissen will, wie es denn wirklich gewesen ist in 
signifie ne considérer la littérature que dans son aspect dénotatif. Et c'est bien là, semble-t-il, la stratégie adoptée par nombre de commentateurs du livre, mais aussi pour l'adaptation faite par Thomas Kirchner ${ }^{21}$. En effet, l'incipit du roman disparaît totalement de l'adaptation télévisée de même que la série des autres chapitres s'y rattachant et qui sont des parties du journal tenu par Meno Rohde, l'un des protagonistes du roman. En supprimant tous ces moments, Thomas Kirchner supprime du même coup la technique du contrepoint, l'une des caractéristiques du roman, et dont la fonction réside dans un éclairage de la diégèse par l'un des personnages qui tente dans le même temps de s'y soustraire. De plus, le titre donné à ce premier chapitre, Ouverture, n'est pas sans importance puisque toute une partie de la famille Hoffmann est mélomane, joue d'un instrument, donne des concerts de chambre. Le thème musical est ainsi, dès le début, indissociablement lié au personnage de Meno et à l'image qu'il dessine du cadre de l'action - le second paragraphe est d'ailleurs tout entier consacré à des réflexions d'ordre musical. L'opposition mise en place dans ce premier chapitre entre le fleuve, son mouvement, sa couleur, sa fonction d'artère économique et d'exutoire d'une part et de l'autre le temps qui organise la vie des hommes, structure leur histoire et semble ralentir inlassablement, comme pour figer ce pays dans un sommeil de Cendrillon, ne se retrouve donc plus dans le téléfilm. Toutes les connotations d'écoulement - du fleuve et du temps -, de ramifications des bras du fleuve, du bruit des horloges, du caractère éternel de l'élément aquatique qui n'existent plus dans la production télévisée ôtent une des épaisseurs au récit :

En quête, le fleuve semblait se raidir dans la nuit naissante, sa peau se froissait et crépitait ; on aurait dit qu'il voulait prendre de court le vent qui se levait dans la ville lorsque la circulation sur les ponts s'était déjà réduite à quelques voitures et à de rares tramways, le vent de la mer enserrait l'Union Socialiste, l'Empire Rouge, l'Archipel, irrigué nourri traversé innervé envahi par les artères les veines les capillaires du vaste fleuve, alimenté par la mer, dans la nuit le fleuve qui ramenait avec lui les bruits et les pensées sur sa surface scintillante, le rire, le sérieux, la gaieté dans la pénombre croissante ; des matières en suspension descendant dans ses profondeurs, où se mêlaient les caniveaux de la ville $[\ldots]^{22}$.

der späten DDR, sollte man ihm rasch und entschlossen den neuen Roman von Uwe Tellkamp in die Hand drücken. »Idem, quatrième de couverture.

21. - La question se pose de savoir s'il ne s'agit pas d'une stratégie marketing de la maison d'édition pour réussir à vendre un roman d'une extrême complexité tant sur le plan stylistique que sur le plan diégétique

22. - « Suchend, der Strom schien sich zu straffen in der beginnenden Nacht, seine Haut knitterte und knisterte; es schien, als wollte er dem Wind vorgreifen, der sich in der Stadt erhob, wenn der Verkehr auf den Brücken schon bis auf wenige Autos und 
Le rythme de la phrase allemande, ce premier chapitre de cinq pages n'étant qu'une seule et même phrase qui se termine sans ponctuation, contribue à renforcer l'image du fleuve et du temps inexorables; seule l'organisation typographique - les tirets qui débutent chaque paragraphe - institue des pauses dans le flot ininterrompu des pensées de Meno. Il y a donc, dans cette ouverture, un véritable stream of consciousness, et les contrastes et oppositions les plus forts que l'on puisse imaginer : d'un côté la langue que rien n'arrête, le caractère inflexible et inhumain de la nature, son rythme tranquille parce qu'éternel, et de l'autre la structure linguistique de la phrase qui semble presque haleter, qui ne permet nulle respiration et produit un sentiment de panique, tout en établissant un rythme proche de l'art de la fugue par la reprise et le croisement de certaines images ou certaines structures de phrases (« suchend, der Strom schien », p. 7 ; « suchend in der Nacht der Strom », p. 10, par exemple). Le mot «Atlantis », en capitales trois fois (p. 9), ajoute une association mythologique au sous-titre et fait de la sphère présentée un monde bien particulier situé à la limite du réel et de l'imaginaire. Ce caractère liminal, ambigu relève bien entendu tout entier de la langue, et l'on voit déjà l'une des raisons qui ont poussé le scénariste à supprimer cette ouverture : comment fixer en images un monde qui oscille entre réalité, fantaisie, fantasme et mythologie?

Le téléfilm, quant à lui, débute par une nuit d'hiver sur un panorama de la ville de Dresde traversée par le fleuve au loin avec, au premier plan, le téléphérique qui monte très lentement ; les cliquetis de la machine couvrent peu à peu la musique du générique, il y a très peu de lumières, le fond est perdu dans la brume du soir. Apparaît, après la liste des producteurs, à la dix-septième seconde, au bas de l'écran : " Dresde 1982 ». Contrairement au roman donc, il y a là un refus de l'ambiguïté et un ancrage géographique et historique précis qui va conditionner le regard du spectateur dans le sens d'un film historico-documentaire, ce que le roman refusait ouvertement dans son épigraphe. Ce changement radical de position est tout à fait symptomatique du désir des commanditaires, qui entendaient ainsi célébrer le jour de l'unité allemande, et du changement d'orientation politique, au sens fort du terme. Alors que le roman se présente comme une réflexion sur un État totalitaire, la vie, les cruautés et les mesquineries auxquelles il contraint ses citoyens, le

vereinzelte Straßenbahnen ausgedünnt war, dem Wind vom Meer, das die Sozialistische Union umschloss, das Rote Reich, den Archipel, durchädert, durchwachsen durchwuchert von den Arterien Venen Kapillaren des Stroms, aus dem Meer gespeist, in der Nacht der Strom, der die Geräusche und Gedanken mit sich nahm auf schimmernder Oberfläche, das Lachen und den Ernst und die Heiterkeit ins sammelnde Dunkel; Schwebstoffe hinab in die Tiefe, wo die Rinnsale der Stadt sich mischten; [...] ». Uwe Tellkamp, Der Turm, p. 7 (trad. p. 11). 
pouvoir de l'imagination qui informe en déformant la réalité, la force de la mise en mots et la subversion possible par la littérature, le film s'ancre fermement dans le genre du témoignage historique et désamorce par là même la force de subversion de l'art qui ne semble être fait que, comme dans une conception triviale de la caméra ou de l'appareil photographique, pour enregistrer objectivement les faits qui passent devant l'objectif ${ }^{23}$. Autrement dit, la réflexion sur le matériau utilisé est absente, trop difficile qu'elle est peut-être pour le grand public un soir de fête nationale ou, comme le fait remarquer Stéphane Benassi, du point de vue de l'écriture, « un projet télévision doit avoir une écriture simple, claire, sans surprise, systématique, le film étant le lieu des recherches et des expériences ${ }^{24} »$. Le mode d'adaptation utilisé serait ainsi choisi pour le roman en question qui ne serait qu'une œuvre testimoniale où la forme et le style n'influeraient en rien sur le message proposé par le texte. Le contexte de réception - 20 ans environ après la réunification - a très certainement été déterminant, mais hélas aussi largement réducteur ${ }^{25}$.

L' ' ouverture » se poursuit avec un jeune garçon assis dans le téléphérique, pratiquement seul dans la cabine qui essuie la vitre du revers de sa manche, visiblement angoissé par la note de 4 à un devoir de mathématiques qu'il tient froissé dans sa poche. Il quitte la station pour

23. - Telle est la teneur de l'article de Peter Zander dans le Berliner Morgenpost du $1{ }^{\text {er }}$ octobre 2012 «Une grande épopée, radicalement épurée » (Ein großes Epos, radikal entschlackt $:$ : [...] Ein so dickes Buch adaptieren, dass alle, die es bewältigt haben, unisono als unverfilmbar klassifizieren? Selbst Drehbuchautor Thomas Kirchner hat lange mit seiner Aufgabe gehadert, dieses unübersichtliche Geflecht von Figuren und Stilmitteln in eine halbwegs überschaubare Dramaturgie zu bündeln. Das ging selbstredend nicht ohne große Auslassungen und schmerzliche Einschnitte. [...]» «[...] Adapter un livre d'une telle ampleur que tous ceux qui ont réussi à le lire jusqu'au bout classent unanimement comme infilmable? Même le scénariste Thomas Kirchner s'est longtemps affronté à sa tâche pour concentrer cet entrelacs intriqué de personnages, de moyens stylistiques en une dramaturgie dont on peut avoir à peu près une vue d'ensemble. Bien entendu cela ne se fit pas sans grandes omissions et douloureuses coupures [...] ».

24. - Stéphane Benassi, op. cit., p. 52.

25. - Voir Jens Binsky, in : Süddeutsche Zeitung (03/10/2012) : « Der Satz, die DDR sei ein Scheiß-Staat, war im Roman plausibel, aber er war nicht die Quintessenz des Buches, das mit einem überbordenden Reichtum an Figuren, Stimmen, Tonlagen, Perspektiven das Bild einer verwunschenen Gesellschaft heraufbeschwor. [...] Ein liebevoll zusammengestellter Bilderbogen [...] als Illustration von Zeitgeschichte, statt von der Fiktion her ein anderes Licht auf Geschehenes fallen zu lassen. » / « La phrase : "la RDA est un État de merde" était plausible dans le roman, mais elle n'était pas à elle seule la quintessence du livre qui évoquait avec une richesse débordante de personnages, de voix, de tons, de perspectives l'image d'une société ensorcelée. [...] Une planche amoureusement constituée [...] comme illustration de l'histoire contemporaine au lieu de jeter depuis la fiction une autre lumière sur ce qui est advenu. » http://www.sueddeutsche.de/ medien/der-turm-in-der-ard-so-wie-sie-waren-1.1485221 (page consultée le 15/07/2013). 
se rendre chez lui, portant bagages à la main et violoncelle sur son dos et croisant un homme en train de réparer sa voiture qu'il salue comme une connaissance, tous les plans montrant l'acteur de face. Le titre «Der Turm Teil 1 » apparaît. Les couleurs sont dans les tons grisâtres, verdâtres et marron, comme la nuit peut très bien les produire. Puis la caméra passe dans le dos de l'acteur pour présenter la rue mal éclairée, traduisant la situation économique alors difficile du pays. À la seizième seconde, le garçon arrive, la caméra le filmant de face à nouveau, et entre dans une grande maison bourgeoise de l'époque du Gründerzeit tout éclairée, entourée d'une longue grille en fer forgée, qui n'est pas sans rappeler la couverture du roman. Au moment où il ouvre la porte, on aperçoit sur celle-ci des branches de sapin avec un ruban rouge, signes de la saison festive de fin d'année. La musique de fond rappelle presque celle de Philippe Glass, mais en beaucoup moins frénétique, tout en étant empreinte d'une certaine menace par la répétition du même thème à intervalles réguliers ${ }^{26}$.

La lecture que le scénariste et le réalisateur ont faite du roman est assez bien résumée par Harald Jähner :

L'adaptation télévisuelle du livre d'Uwe Tellkamp n'a que peu de rapports avec le roman. Seul le squelette de l'action a été recyclé pour le scénario, et encore seulement en partie, disons seuls le squelette et le popotin. Des condensations et des resserrements extrêmes sont inévitables avec un modèle de près de mille pages. Mais que pas la moindre tentative n'ait été faite de transposer ne serait-ce qu'une petite partie des originalités linguistiques du livre dans une langue cinématographique adaptée, voilà qui déçoit ${ }^{27}$.

Ainsi, le côté magique, par exemple des lieux avec les noms des différentes villas et leurs puissantes connotations, n'a été retenu ni par le scénariste ni par le réalisateur comme un élément pouvant être présenté étant donné le présupposé de lecture qu'ils ont décidé d'adopter ${ }^{28}$.

26. - Idem.: « Die Exposition ist grandios gelungen, den Drehbuchautor Thomas Kirchner kann man für seine Virtuosität nur bewundern [...]» / «L'exposition est génialement réussie, on ne peut qu'admirer le scénariste Thomas Kirchner pour sa virtuosité $[\ldots] \gg$.

27. - Harald Jähner, in : Berliner Zeitung (26/09/2012) : « Die Verfilmung von Uwe Tellkamps "Der Turm" hat wenig mit dem Roman zu tun. Für das Drehbuch wurde nur das Skelett der Handlung herauspräpariert, und auch das nur zum Teil, sagen wir gerade mal Gerippe und Gesäß. Extreme Straffungen sind bei einer knapp 1000 Seiten langen Vorlage nicht zu vermeiden. Aber dass nicht der geringste Versuch unternommen wurde, von den sprachlichen Eigenwilligkeiten des Buches einen kleinen Teil in eine adäquate Filmsprache hinüberzuretten, enttäuscht doch sehr. »

28. - Idem. : « Nichts also von dem bohrenden, oft geradezu besessen wirkenden Genauigkeitssinn des Buches, nichts von der auf viele Weisen unwirklichen Atmosphäre, in die Tellkamp das letzte Jahrzehnt der DDR taucht. » / «Rien donc du sens de la précision du livre ni de son effet obsessionnel, lancinant, rien de l'atmosphère irréelle 
Même la question de l'espace, toujours fragmenté, divisé, réduit, que ce soit dans la Maison aux mille yeux ou à la Caravelle, que ce soit chez Josta ou Judith Schevola, dans les lieux et les administrations publics : ce sentiment de claustrophobie ne semble pas représenté si fortement dans la version pour la télévision. Certes, les maisons et leur intérieur sont minutieusement filmés, mais plus dans un souci d'exactitude historique que dans une volonté de restituer cette claustrophobie omniprésente dans le roman, sauf peut-être pour l'appartement occupé par Judith Schevola dans la seconde partie.

La première partie se termine au bout d'une heure vingt-sept minutes par une fête chez les Hoffmann, interrompue par la sonnerie du téléphone. Richard décroche et l'infirmière en chef lui apprend la tentative de suicide de Josta. Après un moment de choc, il retourne à la fête, Anne venant le chercher. La seconde partie - après avoir rappelé par des morceaux de scène la trame narrative de la première partie s'ouvre à la cinquante-cinquième seconde sur le ciel de Dresde au lever du jour, par temps de brouillard, à moins qu'il ne s'agisse de nuages dus à la pollution, et sur l'envol d'un groupe d'oiseaux, symboles de liberté et d'évasion que la caméra suit pendant vingt secondes avant qu'ils ne disparaissent derrière les toits 29 . À une minute zéro cinq apparaît au bas de l'écran : « Dresde 1983 », et Richard regarde à la dérobée Josta emmener ses enfants à l'école. C'est un moment visiblement difficile pour lui, mais en même temps il pousse finalement un soupir de soulagement de voir qu'elle se porte bien. Le titre « Der Turm Teil 2 » apparaît alors sur la façade de l'immeuble devant lequel passe Richard. La décision de couper le roman en épisodes chronologiquement identifiables, même s'il fait sens, interrompt précisément de manière répétée le flux et le flot des images, alors qu'une absence de chronologie explicite aurait conservé quelque chose de la métaphore d'entrée du roman et de la magie d'un temps qui s'écoule à un rythme qui lui est propre. La longueur de la première partie s'explique par la mise en place du contexte politique, social, familial, émotionnel dans lequel évoluent les personnages, la présentation des relations qui les unissent. Après cela, l'histoire peut vraiment commencer. Cet usage particulier qui est donc fait du temps ne correspond que partiellement à ce qui se produit dans le roman, la première moitié se terminant sur la rupture de Josta avec

à bien des niveaux dans laquelle Tellkamp plonge la dernière décennie de la RDA. »

29. - Cette envolée d'oiseaux sera, sous forme d'écho, reprise dans le plan final du téléfilm, qui fait signe vers la conquête de la liberté et le nouveau départ que la fin de la RDA semble promettre. D'ailleurs le téléfilm ajoute une coda totalement inexistante dans le roman : Annette et Meno viennent chercher Christian à sa sortie de la caserne pour rentrer à la maison, mais celui-ci leur annonce son désir de vouloir vivre seul et commencer sa vie nouvelle en adulte indépendant. 
Richard. Malgré ces différences, certains critiques, comme Andreas Platthaus, félicitent et louent le scénariste pour le travail accompli ${ }^{30}$. En effet, le scénariste aurait compris à quelle tâche impossible il s'est voué et décidé très logiquement de recentrer le film sur le drame psychologique des personnages principaux. Ce faisant il se donnait la possibilité d'exprimer et de montrer, à travers les drames de la famille Hoffmann comme exemple d'une certaine couche de la société de la RDA, toutes les dernières années de ce pays :

La manière dont il a dû - malgré les trois heures que dure le téléfilm - réduire les mille pages de La Tour et malgré cela mis au centre des thèmes qui sont cachés ou sous-développés dans le roman, voilà qui traduit une profonde compréhension de la différence entre narration littéraire et narration filmique. [...] Kirchner a un sens inné de ce qui peut être raconté par des regards, des gestes, des constellations, brefs des moyens optiques. De la même manière qu'Uwe Tellkamp en a pour ce qui ne peut qu'être décrit ${ }^{31}$.

Les jeux de mimiques, les échanges de regard, les coups d'œil rapides sont effectivement très présents dans le film, en particulier dans la scène de mariage où Richard se trouve confronté à Josta, où Anne croise ces regards dérobés, où Christian jette un regard attristé et désabusé sur le monde dont il est issu - autant de possibilités utilisées par le scénariste pour rendre des états psychologiques que seuls les mots pourraient sinon décrire. Ainsi le recentrage de l'action sur le personnage de Richard et sur sa double vie, qui semble avoir scandalisé quelques critiques, offre la possibilité d'intensifier, de donner de la substance à des personnages qui sinon en manqueraient peut-être et de montrer un aspect méconnu de la vie en RDA. Les scènes de rue, des manifestations, ont en revanche toutes un air de déjà-vu qui n'apporte rien à l'histoire et à l'Histoire. La décision de Thomas Kirchner et de

30. - Andreas Platthaus, in : FAZ (03/10/2012) : « Ein Triumph der Adaptation. [...] Das Ergebnis ist eine jener seltenen Sternstunden des Fernsehens. » / « Un triomphe de l'adaptation. [...] Le résultat d'une de ces rares heures de gloire de la télévision ». On notera d'ailleurs que ce même auteur avait remarqué, lors de sa recension du roman dans le même journal « Die Zeit ist des Teufels » FAZ (14/10/2008) : « [...] dies ist ein synästhetischer Roman, der alle Sinne fordert, der einen das Sehen und Hören lehrt und gleichzeitig Hören und Sehen vergehen lässt in der Dichte seiner Beschreibungen [...] » Andreas Platthaus remarque ainsi déjà une certaine proximité du roman au média cinématographique.

31. - Jens Binsky, art. cit. : "Wie er die tausend Seiten "Turm" notgedrungen (trotz insgesamt drei Stunden Sendezeit) verknappt hat und trotzdem etliche Motive, die im Buch nur versteckt oder gar nicht ausgebreitet werden, in den Mittelpunkt rückt, das beweist ein profundes Verständnis des Unterschieds zwischen literarischer und filmischer Narration. [...] Kirchner hat ein Gespür für das, was durch Blicke, Gesten, Konstellationen, also optische Mittel, erzählt werden kann. Genauso wie Uwe Tellkamp ein Gespür hat für das, was man nur beschreiben kann. » 
Matthias Schwochow de confier à Richard Hoffmann le rôle principal est un choix qui se justifie et permet d'ordonner la diégèse sans à-coups et de manière continue dans une chronologie précise ; la division par dates permet également de montrer la progression du pays vers la catastrophe en parallèle à la détresse et aux impasses psychologiques dans lesquelles s'enferme le personnage et qui le mèneront dans un asile psychiatrique d'où il sortira dans les dernières minutes du film. On comprend très bien que le « couple » du roman - Meno et Christian soit remplacé par celui du père et du fils, cela leur confère une présence accrue et plus approfondie psychologiquement, renouant aussi par là avec la question générationnelle qui n'a pas cessé d'être au cœur des ruptures de l'histoire allemande du $\mathrm{Xx}^{\mathrm{e}}$ siècle.

Le film Der Turm relève d'une conception particulière de son réalisateur quant à la fonction et à l'essence du cinéma, grand ou petit écran, et qui semble n'avoir envisagé le film que sous l'angle « effet de réel » ou « effet de réalité ». Ce faisant, le texte adapté subit par contrecoup une dévalorisation conceptuelle et factuelle. Ce procédé rend incapable de concevoir la complexité du médium littéraire mais aussi, par ricochet, celle du médium cinématographique. Loin de vouloir condamner par principe l'adaptation littéraire au cinéma, il nous a semblé plus judicieux de chercher à comprendre les enjeux des deux média, ce que le réalisateur de Der Turm mais, au vu des témoignages, étrangement aussi le romancier ne semblent pas à même de faire. Le recadrage de l'action sur le couple père-fils donne au film un nouveau centre névralgique qui réorganise l'action de manière psychologisante. Enfin, il s'agit aussi de toute une vision, par les Allemands eux-mêmes, de la période qui mena à la réunification du pays; et la manière dont ils tentent à l'heure actuelle, avec la distance que le temps octroie, de donner un statut à cet événement historique dans leur imaginaire collectif est révélatrice des choix d'adaptation effectués. La force du média moderne et populaire de grande écoute qu'est la télévision permet, pour des moments de célébration collective, de confectionner des produits très bien calibrés et correspondant aux attentes du public, car les fictions télévisuelles ont un statut différent de la littérature, en termes de production, de discursivité et de rapport au référent, de telle sorte que, comme l'écrit John Corner,

Les fictions populaires sont un vecteur de savoir social, un répertoire de catégories mentales ${ }^{32}$.

32. - John Corner, op. cit., p. 121. 